\title{
ELECTRONIC STRUCTURE OF METALS AND ALLOYS BY $a b$ initio METHODS
}

\author{
Andrzej Jezierski \\ Institute of Molecular Physics ,Polish Academy of Sciences, \\ Smoluchowskiego 17, 60-179 Poznań \\ e-mail : jeziersk@ifrnpan.poznan.pl
}

\section{Introduction}

The electronic structure of metals and alloys was studied in the last years by the different ab-initio methods. The band structure of the ordered systems can be calculated by LMTO, KKJR or APW methods. The Linear Muffin Tin Orbital (LMTO) method is the linearized version of the Kornnga-Kohn-Rostoker (KKR) method [1], This method is very fast and its formalism is simple and transparent. Tire LMTO method can be used on the different types of computers without any problems. The band structure for the simple systems can be computed on the IBM PC 486 computers. For the systems with the many atoms per cell the band calculations are made by using the faster computers (IBM RISC 6000, HP 735, SGI or Cray). The electronic structure of the disordered systems is computed by the coherent potential approximation (CPA) and KKR or LMTO methods. The band structure, the total energy and the thermodynamics properties give the possibility to estimate the phase diagrams of the new systems.

\section{The Linear Muffin Tin Orbital method ( L M TO)}

In the LMTO method [1] in the Atomic Sphere Approximation (ASA) the Wigner Seitz cell is replaced by sphere with the radius $S_{W S}$. The value of the radius of sphere is determined by the condition $\sum_{\mathrm{t}} \mathrm{S}_{\mathrm{t}}{ }^{3}=\mathrm{NS}_{\mathrm{WS}}{ }^{3}$, where the summation is over all $\mathrm{N}$ atoms in the cell. In the ASA the spheres filled completely tire space. The linear muffintin orbital (MTO) is defined by the solution of the one-electron Schroedinger equation and has the form [1] .

$$
\chi\left(r_{R}\right)=\phi_{R L}\left(r_{R}\right)+\sum \phi_{R L}^{*}\left(r_{R}\right) h_{R L . R L}
$$

where $\phi_{R L}\left(r_{R}\right)$ is the solution of the radial Schroedinger equation for the spherical one electron potential $\mathrm{V}(\mathrm{r})$ and $\phi_{\mathrm{RL}}{ }^{*}\left(\mathrm{r}_{\mathrm{R}}\right)$ is the energy derivative function for $\varepsilon=\mathrm{E}_{\mathrm{v} \mathrm{RL}}$ 
The matrix element $h_{\text {RL. }{ }^{\prime} L}{ }^{\prime}$ is determined by the potential parameters $C$ and $\Delta$ and the screened structure matrix $\mathrm{S}$,

$$
\mathrm{h}_{\mathrm{RLR} \mathrm{R}^{\prime}}=\left(\mathrm{C}_{\mathrm{RL}}-\mathrm{E}_{\mathrm{v}^{\prime} \mathrm{RL}}\right) \delta_{\mathrm{RR}} \cdot \delta_{\mathrm{LL}} \cdot+\Delta_{\mathrm{RL}}{ }^{1 / 2} \mathrm{~S}_{\mathrm{RL} \mathrm{R}^{\prime} \mathrm{l}} \cdot \Delta_{\mathrm{R} \mathrm{L}^{\prime}}{ }^{1 / 2}
$$

The potential parameters $C_{\mathrm{RL}}$ and $\Delta_{\mathrm{RI}}$ are defined by $\phi_{\mathrm{RL}}$ and $\mathrm{E}_{v \mathrm{RL}}$ is the middle

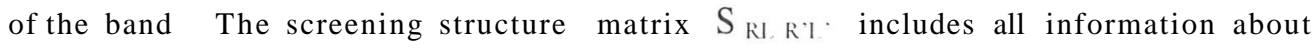
the distribution of atoms. For the crystal the wave function is the linear combination of the partial wave functions

$$
\Psi_{j}(r)=\sum \chi\left(\varepsilon_{j}, r_{R}\right) u_{R L . j}
$$

and the secular equation has the form [1],

$$
\sum\left[P_{R^{\prime} L} \cdot(\varepsilon) \delta_{R^{\prime} L \cdot R L}-S_{R L R \cdot L}\right] u_{R L j}=0 .
$$

In the linear approximation the potential function $\mathrm{P}(\varepsilon)$ can be written a

$$
\mathrm{P}(\varepsilon)=(\varepsilon-\mathrm{C}) / \Delta
$$

hence we got the system of the linear equations

$$
\sum\left[\mathrm{H}_{\mathrm{RL} \cdot \mathrm{R}^{\prime} \mathrm{L}^{\prime}}-\varepsilon \mathrm{O}_{\mathrm{RL} \cdot \mathrm{R}^{\prime} \mathrm{L}^{\prime}}\right] \mathrm{u}_{\mathrm{RL} \cdot \mathrm{j}}=0
$$

where

$$
\mathrm{H}_{\mathrm{RL} \cdot \mathrm{R}^{\prime}{ }^{\prime}}=\mathrm{C}_{\mathrm{RL}}, \delta_{\mathrm{RR}}, \delta_{\mathrm{LL}}+\Delta_{\mathrm{RL}}{ }^{1 / 2} \mathrm{~S}_{\mathrm{RL} \mathrm{R}^{\prime} \mathrm{L}} \cdot \Delta_{\mathrm{R}{ }^{\prime} \mathrm{l}}{ }^{1 / 2}
$$

and

$$
\mathrm{O}_{\mathrm{RL} . \mathrm{R}^{\prime}{ }^{\prime}} \cdot=\delta_{\mathrm{RR}} \cdot \delta_{\mathrm{LI}}
$$

\section{Coherent Potential Approximation ( C P A )}

The electronic structure of the disordered alloys can be calculated by the coherent potential approximation (CPA). In the CPA we replace the real alloy by the effective system defined by the complex coherent potential $\sigma_{L}(z)$. The value of $\sigma_{L}(z)$ is determined from the condition of vanishing of the configurational average of the scattering matrix $\mathrm{t}$,

$$
<\mathrm{T}>=\sum \mathrm{c}_{1} \mathrm{t}_{1}=0
$$

where $c_{1}$ denotes the concentration of the 1-atom 
In the single-site approximation the equation (9) has the

$$
t_{1}=\frac{P_{L}(z)-\sigma_{L}(z)}{1+\left[P_{L}(z)-\sigma_{L}(z)\right] \Phi_{L}(z)}
$$

where $P_{L}(z)$ is the potential function and $\Phi_{L}(z)=N^{-1} \sum[\sigma(z)+S(k)]_{L L}{ }^{-1}$

$\mathrm{S}(\mathrm{k})$ is Fourier transform of the screened structure matrix $\mathrm{S}$.

The local density of states is defined by the imaginary part of $\Phi_{\mathrm{L}}(\mathrm{z})$ function.

$$
\rho_{l}^{\mathrm{Q}}(\mathrm{E})=-\mathrm{P}_{\mathrm{l}}^{\mathrm{Q}}(\mathrm{E}) \operatorname{Im} \Phi_{\mathrm{L}}{ }^{\mathrm{Q}}(\mathrm{E}+\mathrm{i} 0) / \pi
$$

and the total DOS equals

$$
\rho(E)=\sum_{1} \sum c^{Q} \rho_{l}^{Q}(E)
$$

here $\Phi_{\mathrm{L}}^{\mathrm{Q}}(\mathrm{z})=\Phi_{\mathrm{L}}(\mathrm{z}) /\left\{1+\left[\mathrm{P}_{\mathrm{l}}^{\mathrm{Q}}(\mathrm{z})-\sigma(\mathrm{z})\right] \Phi_{\mathrm{L}}\right\}$

4. Electronic structure of ordered and disordered $\mathrm{Pd}_{2} \mathrm{TiSn}$ alloys

The electronic properties of PcbTiSn Heusler type alloys depend on the type of ordering We present the band structures of ordered and disordered Pd2TiSn alloys obtained by LMTO [1] and LMTO-CPA [2] methods. The self-consistent spinpolarised LMTO calculations have shown that these systems were non-magnetic. In the band calculations we assumed the initial configurations according to the periodic table of elements. We applied the atomic sphere approximation (ASA) in which the volume of the Wigner-Seitz cell was replaced by the sphere with the same volume. The electronic structures for the Pd2TiSn was computed for the experimental lattice parameter. In Fig. 1 we present the total density of states (DOS) for the ordered Pd2TiSn alloy The Fermi level is located at $\mathrm{E}=0 \mathrm{eV}$. The DOS consists of three parts. The lower part gives the contribution from s- states of Sn. The middle part contains the d-states of Pd, however near the Fermi level are located the d-states of Ti. The self-consistent spin-polarised band calculations based on the LMTO-ASA and the Local Spin Density Approximation (LSD) indicated that there was no magnetic moment neither on Ti nor on Pd atom. The plot of the total DOS for Pd2TiSn is similar to UPS spectra for Pd2TiAl [3] (solid line in Fig. 1). In many Heusler type alloys the electronic and magnetic properties depend on the local ordering. The effect of the disorder was studied in the terms of the coherent potential approximation (CPA). 


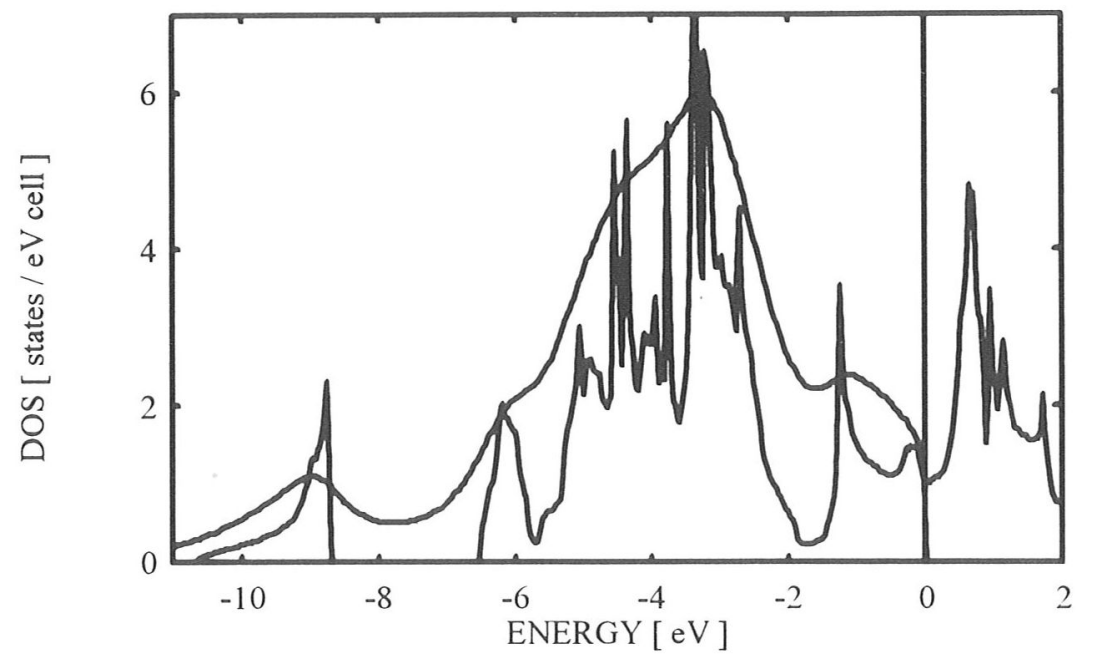

Fig. 1 The total density of states for ordered $\mathrm{Pd}_{2} \mathrm{TiSn}$ alloy.

We have calculated the electronic density of states for the disordered Pd2TiSn alloy using the LMTO- CPA method [2]. In Fig.2 the total DOS for Pd2TiSn disordered alloy is plotted. The Fermi energy is located at $\mathrm{E}=0 \mathrm{eV}$. We observe peaks in the similar position as in the ordered systems The disordered Pd2TiSn is also non-magnetic system. In the disordered system we do not observe the gap between s-states of Sn and d-states of Pd.

\section{Conclusions}

The self-consistent ab-initio LMTO and LMTO-CPA methods give the possibility of calculation the electronic structure of ordered and disordered metallic systems.

We can also study the effect of local environment by using the supercell with the large number of atoms. In such supercell we can change the positions of atoms and made the ab-initio calculations for the large unit cell The LMTO method is very fast and it can be used on the different kind of computers. 


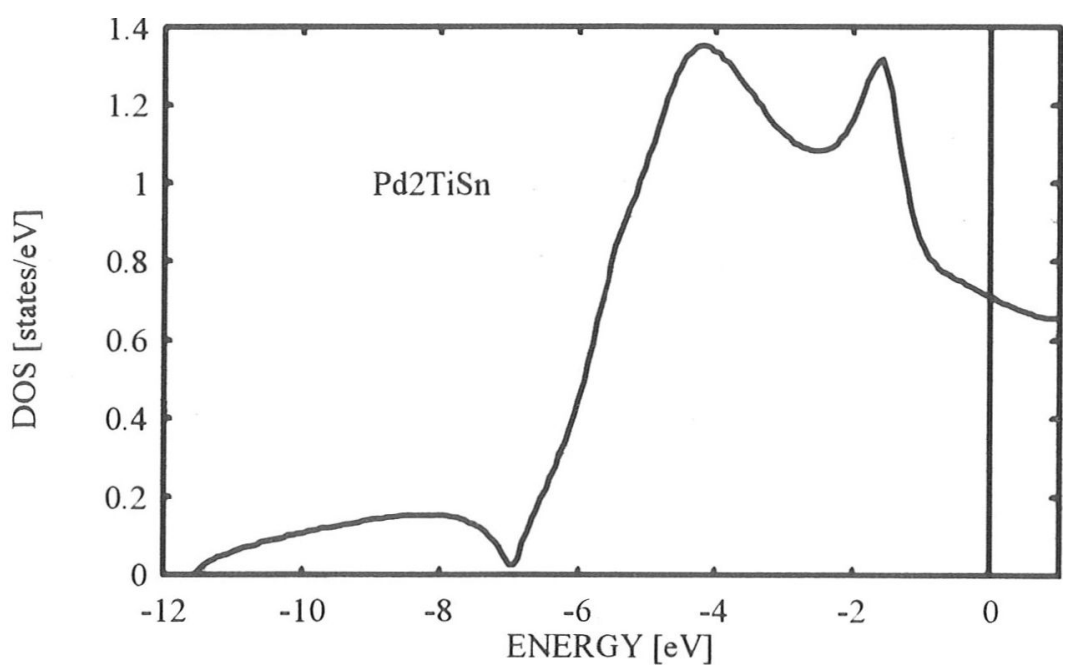

Fig. 2 The total density of states for the disordered $\mathrm{Pd}_{2} \mathrm{TiSn}$ alloy.

This research was supported by the State Committee for Scientific Research through the Project No 8T1 IF 01008 p04

\section{REFERENCES}

[1] O.K. Andersen ,0. Jepsen and M.Sob, in: Electronic Structure and Its Applications (ed.. Yussouff(Berlin-Springer) p. 2 (1987).

[2] J.Kudrnovsky and V.Drchal, Phys.Rev.B.41, 7515 (1990).

[3] A.J.Liddiard, M.D.Crapper, N.D.TellingJP. A.Skull, M. Petty,N.K.Zayer, J.G.Smith, K-U.Neumann and K.R.A.Ziebeck, J.Magnetism magnetic Mater. 140-144,187 (1995). 